\title{
SISTEMATIZACIÓN, UNA PRÁCTICA NECESARIA
}

\author{
SYSTEMATIZATION, A NECESSARY PRACTICE
}

Máryuri García González

\section{Resumen}

En el trabajo se realizan análisis de la sistematización como proceso social desde la educación popular, sus enfoques, tendencias e importancia para el buen desarrollo de los proyectos locales. Se realiza además un abordaje teórico que nos permite reconocer la importancia de la sistematización en estos proyectos para el desarrollo individual y grupal desde la contextualización de Freire. Se tienen en cuenta los elementos objetivos y subjetivos para el proceso de sistematización y su influencia en el desarrollo local y comunitario de manera competente.

Palabras claves: Sistematización; Proceso; Desarrollo local; Educación popular.

\section{Abstract}

In work carried out systematic analysis of the social process from popular education, approaches, trends and importance for the proper development of local projects. It also takes a theoretical approach that allows us to recognize the importance of systematizing these projects for individual and group development from the contextualization of Freire. It takes into account the objective and subjective elements to the systematization process and its influence on local development and community competently.

Keywords: Documentation; Process; Local development; Popular education.

\section{Análisis General desde la Sistematización...}

... "Sistematización, es una propuesta basada en otro paradigma, un paradigma de construcción de los actores, los sujetos, los hombres y las mujeres como transformadores de la historia".

Oscar Jara

Avanza un nuevo tiempo y con ello el reto de transformar estructuras sociales, construir y contribuir a este reclamo y con él la gestación del hombre nuevo, de un sujeto capaz de tributar a la justicia social, lo cual implica comprender y aceptar la diversidad multi y pluricultural que nos compone y con ello las formas de aprendizaje.

Hacer presente las ideas de Paulo Freire, Carlos Núñez, Oscar Jara, concebir la educación como una acción política y de resistencia, cuyo último objetivo es la construcción de una conciencia crítica.

\footnotetext{
${ }^{1}$ Doctora en Ciencias de la Educación. Profesora de la Universidad de la Habana, Cuba. E-mail: maryuri@cepes.uh.cu / maryurigarciagonzales@gmail.com ORCID: http://orcid.org/0000-0002-2734-6541
} 
Tengamos en cuenta entonces el proceso de sistematización desde la educación popular como la reconstrucción de la experiencia vivida para analizar la práctica y considerar esta como un nuevo punto de partida, desde y para la acción de transformación protagonizada por los propios sujetos con carácter participativo.

Lo metodológico es clave para el proceso de sistematización, pero no es común que se reconozca su complejidad en sentido profundo; lo que implica sustentar teóricamente y organizar de manera rigurosa la secuencia de momentos de manera coherente con la fundamentación teórico - filosófico y que se ejecute de forma creadora, participativa y por supuesto, teniendo muy en cuenta las particularidades del contexto, características y distinciones de cada experiencia a sistematizar.

Para sistematizar no existe una "receta", depende básicamente de la propuesta y de los coordinadores o facilitadores del proceso de sistematización en sí.

Las diferencias encontradas en cada propuesta, a partir de lo metodológico, tienen que ver con diferencias en su concepción, en los objetivos previstos, los objetos a sistematizar, los ejes a trabajar de acuerdo a las necesidades de la sistematización, de las experiencias prácticas de quienes conducen y formulan las propuestas, depende además de factores externos, objetivos y subjetivos que inciden de alguna manera en la sistematización.

Lo metodológico, entonces, es considerado un aspecto fundamental sobre el que es necesario trabajar, profundizar y avanzar, siguiendo siempre la experiencia acumulada en los "encuentros y desencuentros" de la sistematización.

Entre los elementos a tener en cuenta en un proceso de sistematización están incluidos los objetivos y subjetivos:

- Los sujetos: las diversas personas protagonistas de la práctica.

- Los contextos: lugares, espacios donde se desarrolla la práctica, condiciones en que se desenvuelven las prácticas.

- Intereses y motivaciones: el que, porque y para que de las prácticas.

- Referentes: conceptuales, políticos, culturales, económicos, ambientales, sociales; orientar, retroalimentar, acondicionar las prácticas sociales (responder al telón de fondo). 
- Contenidos: información compartida por los sujetos, emociones, sentimientos, motivaciones, ansiedades, aspiraciones que inciden en las relaciones y dinámicas de la práctica.

- Resultados: esperados o inesperados que van surgiendo (cambios, transformaciones de los sujetos, sus relaciones, condiciones sociales a partir de esa práctica.

Nunca la sistematización será:

- Escribir o describir una práctica.

- Recopilar información y ordenarla cronológicamente.

- Enunciar aciertos y desaciertos.

- Evaluación o investigación.

La sistematización posibilita comprender el desarrollo de la experiencia, el cómo y el porqué, así y no de otra manera, cuáles fueron los cambios producidos, como y porque se produjeron, esto nos permite sobre todo entender la relación entre las distintas etapas de un proceso, diferenciando los elementos constantes de los ocasionales, y los que quedaron sin continuidad, los que ocasionaron nuevas líneas de trabajo.

Este proceso permite entender como llegamos al momento en que estamos para comprender mejor nuestro presente y mejorar la práctica y hacerla más coherente en el futuro, superando vacíos, reafirmando las fortalezas, para no repetir debilidades.

Como plantea Jara (2007) puede entenderse sistematización como una conceptualización de la práctica, que le da coherencia a todos sus elementos. De una forma nos acercamos a la comprensión de las diferentes etapas vividas, adentrándonos en sus propias dinámicas y circunstancias, desde el inicio hasta los resultados posteriores.

El proceso de sistematización por tanto permite:

- Evaluar la práctica objeto de sistematización.

- Ordenar información.

- Diseñar indicadores.

- Retroalimentar las prácticas con visión sistémica y sistemática, intencionada. 
- Capacitar recursos humanos.

- Ordenar el nuevo conocimiento.

- Comunicar los aprendizajes y socializarlos.

- Intercambiar las experiencias y aprendizajes.

- Empoderar a los sujetos que realizan la sistematización.

También se hace necesario que los sujetos que tienen a su cargo el proceso de sistematización sean:

- Individuos que participen de la experiencia.

- Personas que asesoren, apoyen y faciliten este proceso (flexibles y no resistentes al cambio)

- Que tengan presente los diferentes roles de los que participan en la experiencia de sistematización (relator, informante, de apoyo, entre otras)

- Habilidad para hacer análisis y síntesis.

\subsection{SURGE LA PREGUNTA ¿QUÉ ES SISTEMATIZAR?}

La sistematización puede considerarse como un ejercicio referido a experiencias prácticas concretas, puede aplicarse a cualquier experiencia que se desee o se necesite, sin embargo, la mayor difusión y aplicación del proceso se enmarca en experiencia de Educación popular, Organización Popular y Proyectos Socio culturales comunitarios.

Básicamente estas experiencias, a partir de criterios de Jara (2007); son procesos dinámicos en permanente cambio y movimiento. Son también procesos sociales complejos, en los que se interrelacionan, de forma contradictoria, un conjunto de factores objetivos y subjetivos:

- Las condiciones del contexto en que se desenvuelven.

- Situaciones particulares a las que enfrentarse.

- Acciones intencionadas que buscan lograr determinados fines.

- Percepciones, interpretaciones e intenciones de los distintos sujetos que intervienen en ellos. 
- Resultados esperados e inesperados que van surgiendo.

- Relaciones y reacciones entre las personas que participan.

Son procesos además particulares, que hacen parte de una práctica histórico social general, de igual manera dinámica, compleja, contradictoria y pudiera decirse que trasformadora.

Desde los criterios de Álvarez (2007), sistematizar es un proceso de reflexión orientado por un marco de referencia y con un método de trabajo que posibilita organizar un análisis de la experiencia; dar cuenta de lo realizado, facilitar la comunicación y la interpretación de las acciones ejecutadas y la elaboración teórica de las vivencias para iluminar y revolucionar nuevas vivencias.

Todas y cada una de las experiencias que se sistematizan están cargadas de una riqueza acumulada invaluable, de una vitalidad propia y distintiva, de elementos que representan procesos únicos, irrepetibles, propios y dotados de un "genoma" particular e inédito.

De ahí que el proceso de sistematización nos exige tanto de cada uno de nosotros, al ser un proceso apasionante y en el cual es necesario responsabilidad y compromiso para con la tarea, siendo además imprescindible comprender que se quiere y hacia donde se va, extraer enseñanzas y comunicarlas, recuperar lo vivido desde una mirada crítica y reflexiva, siendo capaces de:

- Socializar.

- Intercambiar experiencias.

- Resolver conflictos y trasformar realidades.

- Fortalecer las identidades colectivas.

- Remirarnos para ver quiénes somos y reconfigurarnos como hombres y mujeres bio-sico-sociales.

- Recuperar lo vivido en aras de ganar fortalezas y empoderamiento.

- Transformar la realidad a la vez que nos transformamos como sujetos desde nuestras miradas, subjetividades y experiencias.

Teniendo en cuenta siempre que "No se sistematiza lo que no se vive", no se sistematiza si no hay confianza, motivación, interés y respeto, porque sistematizar implica "desnudarnos", volver a vivir, sentir y resinificarnos. 
La sistematización como proceso no tiene un concepto o definición única o acabada, existen tantas definiciones como personas tratan el tema, sin embargo, todos tienen puntos coincidentes.

Entre las cuestiones abordadas por diferentes teóricos del tema: Martinic (1984) y Martinic y Walker (1987); Ibañez (1991); Antillón (1991); Colectivo de autores (1991); Jara (2007); Álvarez (2007); Hernández y Pablos (2013) entre otros, se encuentran esencias distintivas por las cuales indistintamente reconocen y acentúan la sistematización:

- Por la reconstrucción ordenada de la experiencia. Por su carácter de proceso productor de conocimientos.

- Por su reconocimiento como proceso participativo.

- Por su contextualización desde lo teórico, metodológico y práctico en la realidad social, política, económica, cultural y su posterior retroalimentación.

- Por la conceptualización de la práctica, para darle coherencia a todos sus elementos.

Coincidimos de alguna manera con los diferentes abordajes teóricos, pero reconocemos que aún falta precisar lo propio dentro del tipo de reflexión o conceptualización de la sistematización. Lo más característico de la reflexión es que busca penetrar en el interior de la dinámica de las experiencias, llegando así a entender estos procesos desde su propia lógica, extrayendo como dijera Oscar Jara, las enseñanzas que pueden aportar al enriquecimiento tanto de la práctica como de la teoría.

La sistematización Jara (2007), es aquella interpretación crítica de una o varias experiencias, que, a partir de su ordenamiento y reconstrucción, descubre o explicita la lógica del proceso vivido, los factores que han intervenido en dicho proceso, cómo se han relacionado entre sí, y por qué lo han hecho de ese modo.

Continúa abordando Jara (2007) que esta afirmación contiene sintéticamente afirmaciones particulares:

- Define la sistematización como interpretación crítica, es decir, como el resultado de todo un esfuerzo por comprender el sentido de las experiencias, tomando distancia de ellas. 
- Solo es posible si previamente se ha ordenado y reconstruido el proceso vivido en esas experiencias.

- Se caracteriza por descubrir la lógica con la que ese proceso se lleva a cabo, cuáles son los factores que intervienen en él y las relaciones entre ellos.

Por tanto, sistematizar no es narrar y clasificar experiencias por categorías comunes, descubrir procesos, ordenar y tabular información sobre experiencias, hacer una disertación teórica ejemplificando con referentes prácticos. La sistematización se sitúa en un intermedio entre la descripción y la teoría, un terreno por el cual se tiene poca costumbre de transitar.

Para sistematizar es necesario pensar de manera dinámica, rigurosa, procesual, crítica, creativa, reflexiva y coherente.

Al sistematizar, como planteara Martinic y Walker (1987) no solo se pone atención a los acontecimientos, a su comportamiento y evolución, sino también a las interpretaciones que los sujetos tienen sobre ellos. Se crea así un espacio para que esas interpretaciones sean discutidas, compartidas y confrontadas.

\section{2. ¿Para qué Entonces Sistematizar? Y ¿Qué Condiciones Debemos Tener en Cuenta para ello?}

Básicamente coincidimos con Álvarez (2007), al plantear que para Sistematizar es necesario:

- Ordenar procesos, acciones y actividades

- Recuperar, resignificar, comprender, evaluar y valorar creativamente una prácticas o experiencias

- Posibilitar un conocimiento más profundo de la realidad

- Contextualizar: desde lo teórico y metodológico y en la realidad social, política, económica, cultural, ambiental, entre otras

- Retroalimentar: evaluar, investigar, cambiar y mejorar (teórica, metodológicamente)

- Formular nuevos conceptos acerca de la realidad 
- Hacer una historia o analogía de la experiencia

- Comparar, intercambiar, comunicar y/o diseminar la experiencia.

Donde sus principales objetivos son: Ordenar, recuperar, mejorar, aprender y comunicar experiencias o prácticas educativas, socioculturales, comunitarias, entre otras; posibilitando mayores intercambios, interacciones, enriquecimiento de la práctica y vínculo con otros proyectos.

La recolección y la organización de la información se realizan, generalmente mediante: Diario de campo, actas, cuestionarios, protocolos, guías de observación colaborativa, portafolio de desempeño, fichas y carpetas temáticas, relatorías, fotos, grabaciones, técnicas utilizadas con los materiales que se generan en ellas, entre otras.

Se complementa con la evaluación, aunque la sistematización es más profunda; ambas identifican logros y dificultades y con ellos se redirecciona y fortalece el trabajo, se hacen ajustes y correctivos, se reflexiona, interpreta y comprende el proceso vivido, analiza desde la teoría, desde la práctica y desde la experiencia desarrollada para producir, conceptualizar o teorizar sobre ella; por lo que se asume la sistematización como un proceso formativo.

Una vez establecido el cronograma de actividades para realizar la sistematización, es criterio de Instituto de Derechos Humanos (2004), que se debe de presupuestar los costos del proceso y disponer de los recursos necesarios; previendo además un tiempo mínimo para poder desarrollar los ejercicios de sistematización y que existan las condiciones para ello, desde el espacio, materiales, instrumentos, entre otras Zilvetty, M.A (2014).

\section{3. ¿Cómo Sistematizar desde la Práctica?}

Es importante al menos desde nuestra experiencia tener presente los siguientes pasos o secuencias:

1. El punto de partida: como factor determinante

- Haber participado en la experiencia

- Tener constatada la experiencia.

2. Interrogantes esenciales 
- ¿Para que? (define el objetivo)

- ¿Qué sistematizar? (define el objeto)

- ¿Qué elemento o eje central nos interesa sistematizar? (eje de sistematización).

3. Recuperación de lo vivido.

- Reconstrucción de la historia.

- Ordenar y clasificar información.

4. Reflexión (¿por qué lo vivido?)

- Analizar, interpretar objetivamente el proceso.

5. Punto(s) de llegada.

- Llegar a conclusiones.

- Comunicar aprendizajes

Desde la perspectiva de Jara (1994) y Jara (2007), es preciso apuntar que la sistematización es un proceso de interpretación crítica como resultado de todo esfuerzo para comprender el sentido de las experiencias, tomando distancia de ellas, solo es posible si:

- Previamente se ha ordenado y reconstruido el proceso vivido

- Caracterizada por describir la lógica con la que ese proceso se lleva a cabo, cuáles son los factores que intervienen en él y la relación entre ellos. Es esta su esencia.

Es oportuno desde la perspectiva de referentes prácticos culturales heurísticos, axiológicos, según Islas (2017), tener presente los objetivos que persigue la sistematización, las cuales pueden resumirse en:

- Comprender: distinguir los elementos esenciales de la práctica cotidiana, interiorizar la misma.

- Interpretar: arribar a conclusiones de las experiencias vividas, ser capaz de analizarlas, relacionarlas.

- Explicar: interpretar, argumentar, ordenar lógicamente, exponer los juicios recopilados.

- Reconstruir: exponer una nueva realidad determinando los criterios relevantes, descubrir sus nexos. 
- Reflexionar: elaborar razonamientos reflexivos a partir del análisis y de los nexos entre los argumentos emitidos.

- Transformar: reconceptualizar, aprehender, reformular experiencias en un proceso dinámico.

Se hace necesario aprender sin embargo que el proceso de sistematización no está exento de incertidumbres tales como:

- En algunos casos es vista como una narración, clasificación de experiencias, descripción de un proceso o como ordenamiento o tabulación de información sobre la experiencia.

- Insuficiente fundamentación sobre herramientas metodológicas y técnicas para realizarla siendo la mayoría de ellas propuestas desde la educación popular.

- Participación de personas ajenas a la experiencia objeto de sistematización, lo cual puede entorpecerla.

- Temor a la confrontación abierta y al debate.

- Las condiciones contextuales en que se desarrolla el proceso de sistematización no siempre favorecen.

- Falta de coordinación en los sujetos que intervienen, lo que provoca relaciones y reacciones dispersas entre los participantes.

- Poca experiencia para asumir este proceso, y procesar la información recopilada.

- El que surjan resultados inesperados que puedan interrumpir el proceso.

Es importante el reconocer la sistematización como un proceso en espiral, como dijera José Martí: "Después de todos los dolores de la vida, que asombro causa verla reducida a unas cuantas verdades y como después de enmarañados sucesos, cuya significación aislada entendidas absolutamente- vemos salir lenta y confusamente sencillas verdades".

En el proceso de sistematización y en aras de que el mismo sea efectivo, lográndose el objetivo propuesto, no podemos dejar de hacernos las siguientes preguntas.

- ¿Para qué se quiere sistematizar? 
- ¿Qué se quiere sistematizar?

- ¿Cómo se realizará la sistematización?

Entonces tengamos en cuenta al respondernos estas preguntas.

1. Fundamentación: breve explicación que argumente el que, porque y para que del proceso de sistematización.

2. Objetivos de la sistematización: explicado de manera sintética el alcance y propósito.

3. Metodología: exponer tareas, métodos, procedimientos que se ejecutarán, las responsabilidades que se asumirá individualmente y compartidas.

4. Recursos: cálculo de tiempo, materiales, recursos logísticos en general para garantizar el éxito de la tarea.

5. Cronograma: planificación adecuada de las tareas del proceso y de lo que se espera obtener como producto (incluye el proceso de recuperación)

También es oportuno destacar que la puesta en práctica del proceso de sistematización requiere tanto de condiciones institucionales y personales que garantizan el éxito de la misma. García (2018).

Entre las condiciones institucionales se pueden destacar.

- Decisión para llevar a cabo este proceso como vía de transformación y como política priorizada, lo que presupone asignación de tiempo, recursos y planificación.

- Creación de un ambiente institucional propicio, teniendo en cuenta que no es solo ejecución sino involucrarse en las decisiones.

En el caso de los facilitadores del proceso:

- Amplia profesionalidad, destreza.

- Compromiso y ética.

- Capaz de comprobar la teoría en sus aplicaciones a la práctica (capacidad y competencia).

Según Jara (2007) para el ejercicio de sistematización son necesarias tres condiciones básicas.

- Interés por aprender de la experiencia. 
- Sensibilidad para dejar hablar a la experiencia por sí misma.

- Habilidad para hacer análisis y síntesis.

No es posible entonces un cambio externo a escala social que antes no pase por lo personal, siendo necesario desde nuestras prácticas de vida buscar una coherencia entre el discurso teórico y práctico. Se hace imprescindible el diálogo desde el compartir los saberes múltiples, para estimular la memoria colectiva, el conocimiento y las prácticas locales, enriqueciéndolas y perfeccionándolas en aras de un espacio mejor para todos.

\section{A Modo de Conclusiones}

Lo que se aspira con la sistematización es el aprovechamiento de las potencialidades individuales y grupales, es que la misma contribuya al mejoramiento de las prácticas, lograr una manera de pensar verdaderamente comprometida, verdaderamente dialéctica.

\section{Referencias Bibliográficas}

Alvarez, M.A. (2007). Sistematizar las prácticas, experiencias y proyectos educativos ¿Tarea del gestor educativo?. Fundación Universitaria Luis Amigó Transversal 51A \#67B 90 Medellín - Colombia. www.funlam.edu.co

Antillón, R. (1991). ¿Cómo entendemos la sistematización desde una concepción metodológica dialéctica?. Documento para discusión. IMDEC- ALFORJA. México.

Colectivo de autores. (1991). Cuadernos de Lectura 2. Sistematización aspectos metodológicos Editorial. CLEBA. Medellín. Colombia. 1991.

García, M. (2018). Sistematización de procesos investigativos. Conferencia impartida en Universidad de El Salvador. Escuela de Postgrado, Facultad multidisciplinaria de Oriente. UES.

Hernández, B y Pablos, R. (2013). Un tejido de muchos puntos. Compilación sobre educación popular ambiental. Editorial Caminos. La Habana. Cuba.

Ibañez, A. (1991). La didáctica en la sistematización de las experiencias. Revista Tarea. Lima. Perú. 1991. 
Islas, M. (2017). Sistematización de experiencias. Curso Metodología del trabajo comunitario desde la Educación Popular. Asociación de Pedagogos de Cuba. La Habana.

Jara Holliday, O. (1994). Para Sistematizar experiencias. Editorial ALFORJA. Costa Rica 1994.

Jara Holliday, O. (2007). Para sistematizar experiencias. Edición Mexicana. IMDECALFORJA. 2007.

Martinic, S y Walker, H. (1987). La reflexión metodológica en el proceso de sistematización de experiencias de educaciçon popular. CIDE. 1987.

Martinic, S. (1984). Algunas categorías de análisis para la sistematización. FLASCO. Editorial CIDE. Santiago de Chile. 1984.

Zilvetty, M.A (2014). Guía para la sistematización de experiencias transformadoras. Gestion- I- 2014. La Paz. Bolivia.

Manuscrito recebido em: 26 de outubro de 2020

Aprovado em: 08 de novembro de 2020

Publicado em: 08 de novembro de 2020 